DOI: $10.20472 /$ TEC.2019.008.028

JONAS VAN WAES

KU Leuven, Belgium

JOAN PEUTEMAN

KU Leuven, Belgium

JENS VANKEIRSBILCK

KU Leuven, Belgium

JULIE VERMEERSCH

KU Leuven, Belgium

WALTER DAEMS

UAntwerpen, Belgium

PETER HELLINCKX

UAntwerpen, Belgium

ENRICO JACOBS

Belgium Campus iTversity, South Africa

JEROEN BOYDENS

KU Leuven, Belgium

\title{
DEMOGRAPHIC IMPACT ON THE QUALITY MANAGEMENT SYSTEM: EUROPEAN GUIDELINES IN A SOUTH AFRICAN CONTEXT
}

\begin{abstract}
:
Since many years, the European Standards and Guidelines are used to assess the quality within Higher Educational Institutions. Due to the increasing international cooperation between universities and university colleges, these standards and guidelines provide an adequate foundation to assess the quality of all potential international partners inside and outside Europe. Due to a mutual desire for collaboration, a group of Belgian academics audited a private Higher Educational Institution in South Africa. Significant demographic differences between Europe and South Africa have a significant impact on the perceived quality. It is the aim of this paper to assess the specific demographic and societal influences which have a direct impact on several student-related processes and the perceived quality of those processes. It is found that a multitude of external factors influence a Quality Management System. More precisely, the impact of the local demographic, cultural, ethnic and economical conditions on the student recruitment, student retention and student employability are considered.
\end{abstract}

\section{Keywords:}

Demographic Impact, Educational Quality, Quality Management Systems

JEL Classification: 124, L15, L31 


\section{Introduction}

The European Community has increasingly become a knowledge-based society. Higher Educational Institutions (HEls) all over Europe want to provide the best educational programmes. This trend is clear in the Engineering Technology institutions, but it appears in other fields of study as well. The concept of the best educational programme is usually measured based on the effectiveness of an institution's Quality Management System (QMS). Following the generic concept of quality as written down in the European Standards and Guidelines (ESG) (ESG, 2015) for QMSs, an assessment of an institution and its programmes can be made. In order to assess institutions and programmes according to the Quality Framework, external quality assurance audits have been conducted for many years (Vermeersch, 2015; Remaud et al., 2017).

Quite recently, a significant rise in international collaborations between universities and industry is noticed. These collaborations are no longer bounded by country borders, nor by distances between continents. Global collaboration is a standard practice, especially when considering research-based education. Active participation in research is mandatory, and research must be carried out in an international and/or global context. It is no longer feasible to have all tools and infrastructure on its own. This is a major difference with pure education-based institutions, where all tools and infrastructure can be provided locally.

Globalisation has, and always will have, characteristic effects on an institution. On the one hand, international collaboration enhances the knowledge and the standard of the educational programmes. On the other hand, research-based educational programmes need to strike a balance between the need to comply with requirements and expectations from (1) the local country, and (2) the international academic world and research field. Known protocols, such as the ESG, mainly focus on the management perspective. This paper claims that this perspective can be too narrow and more emphasis is needed towards the local context. Adaptation to the local context should be part of the QMS, resulting in a direct increase of the impact that HEls have on the local society, economic growth and educational needs. This impact HEls have is significant, and it is paramount that they function properly with an appropriate level of quality (Popa and Moraru, 2019).

By studying the student's educational life-cycle that surpasses the HEI, this paper attempts to fill the void left by the ESG in terms of the demographic influences and considerations. Although these influences are briefly mentioned within the ESG (see Section 2), the authors want to explicitly uncover the different demographic considerations in Europe and South Africa. These differences must be taken into account to have a more adequate QMS.

These findings were the result of an external quality audit performed by the KU Leuven and the University of Antwerp. Their expertise in quality management has been claimed in literature before (Vermeersch, 2015; Remaud et al., 2017). These universities visited a private HEI in South Africa, named Belgium Campus ITversity. This intercontinental audit originated from a mutually desired collaboration envisioned by the institutions. It is important that an audit should be based on a friendly note (Supernak, 2018) and mentioning the positive findings are as important (or even more important) as the negative findings. Furthermore, the audit led to a significant insight in the South African demographic factors that influence an appropriate QMS. The findings and conclusions from the audit provide the running examples in this paper. The contribution of this paper 
is to raise the awareness of different demographic and societal factors. The awareness has an immediate effect to further improve the QMS. An improved QMS leads to improved perceived quality. All stakeholders will benefit from such an approach.

This paper is structured as follows. Section 2 briefly introduces the European Standards and Guidelines with the points of interest to this paper. In Section 3 the methodology of the audit is described. This methodology is important to substantiate the demographic and societal influences described in Section 4. Finally, a number of conclusions are covered in Section 5.

\section{European Standards and Guidelines (ESG)}

The European Standards and Guidelines (ESG) (ESG, 2015) are a set of standards and guidelines for quality assurance in higher education, both internal and external. These ESG are internationally agreed upon, internationally used for quality assurance and updated once every couple of years to stay in touch with the current perspectives on quality assurance. However, these ESG do not specify what quality is, nor do they prescribe how the quality assessment processes should be implemented (ESG, 2015). Within the guidelines, vital areas are covered for successful quality provision and learning environments.

The ESG provide guidelines on a wide range of topics, such as e.g. the quality assurance policies, teaching and student services, etc. However, the ESG focus on quality of the institution from a management point of view. When traversing the ESG with the demographic and societal influences in mind, only a few of the standards provide a link to these important topics. The specific standards found to have a societal link are:

- ESG 1.1 - Policy

"Quality assurance policies are the most effective when they reflect the relationship between research, learning and take account of both the national context in which the institution operates and its strategic approach."

- ESG 1.7 - Information management

"Student progression, success and drop-out rates"

- ESG 1.9 - On-going monitoring and periodic review of programmes

"The changing needs of society"

- ESG 3.5 - Resources

“... highlights a higher education's important impact on the development of societies and individuals ..."

In each of the mentioned standards, the demographic and societal structure has a major impact. Section 4 will attempt to raise the awareness to these demographic and societal impacts and considerations on the QMS. First, the methodology of the conducted audit will be presented.

\section{Methodology of the conducted audit}

The insights described in this paper originate from the audit by a joint external commission of peers from international universities, active in the same study domain as the relevant HEI: Engi- 
neering Technology, with the specific focus of this audit on the Information and Communication Technologies (ICT) programme.

The private South African higher educational institution requesting this audit compiled an extensive self-evaluation report in preparation for the educational assessment (Jacobs, 2018). The commission's on-site visit took place in October 2018. The assessment commission visited the infrastructural facilities of two campuses. Additional documents were provided on site for consultation. These documents included minutes of meetings, policy documents and regulations, a selection of study materials, indications of staff competences, testing and assessment assignments, previous self-evaluation and educational assessment documents, etc. Sufficient time was scheduled throughout the visit to study these documents thoroughly. Additional documentation was requested during the on-site visit when the assessment panel deemed that information necessary to check its findings.

During the visit, the commission interviewed different types of actors involved in the study programmes: management, teaching staff, student support staff, administrative support staff, representatives from the professional field, internationalization partners, researchers, alumni, students and their representatives. These stakeholders have been identified to have a significant impact on the quality of the educational programmes (Vermeersch, 2015). The conversations and interviews took place on site or via Skype (in case of foreign partners) and provided the commission with additions to and clarifications of the information in the self-evaluation report and documents analysed at the start of the visit. The individual panels were composed of stakeholders with a similar profile (e.g. teachers, alumni, students,...). The interviews were conducted in confidence, so stakeholders felt comfortable expressing their point of view. In case one or more interviewees would excessively dominate or influence the dialogue, the commission was able to exclude them from the conversation. Other time slots were consultation time slots, in which the assessment panel could invite further stakeholders on request, or stakeholders could come to the commission on their own initiative.

\section{Demographic and societal influences on quality management systems}

Within this paper, demographic and societal influences are seen in a general extent and go beyond the pure statistical study of populations. Factors affecting the quality management system are complex phenomena, such as language, religion, ethnicity, education and nationality. Alongside demographic factors, other inequalities created by the Apartheid Regime in South Africa are still present: economical factors, the gap between rich and poor (GINI coefficient), educational attainment levels, etc.

All these factors influence the quality management system and therefore the perceived quality of the institution. Multiple external influences can cause a bad score on some of the standards. It is always essential to investigate why a specific decrease in perceived quality is present; i.e. a decrease with regard to the protocol used by the assessment team. It is paramount to check for unknown factors that the quality standards (such as the ESG), developed in a European context, may not have taken into account.

The following subsections will provide an overview of the specific demographic factors to be considered throughout the entire students' study-cycle (or life-cycle within the institution). Note that this paper does not state that in general, bad quality does not exist. However, very often bad 
quality indicators are externally influenced and an appropriate nuance is needed. This paper attempts to provide these nuances and advocates their relevance in the audit.

\subsection{Student recruitment}

Before students can actually be recruited by Higher Education Institutions (HEIs), the students must have the appropriate level of study to even be considered as a potential student. South Africa has a population of around 58 million people. According to South African Market Insights (DHET, 2016), the starting group in Grade 1 was 1.2 million children in 2016. Around 687,000 people made it to Grade 12 in 2016. From those students in Grade 12, only around 400,000 graduate in Grade 12 (DHET, 2016).

Mathematics, alongside life orientation and languages, is a prerequisite for enrolment in all HEls. Recent numbers show that around 172,000 students are eligible to start a bachelor's degree. In 2016, 159,000 first time students enrolled in public HEls in South Africa (DHET, 2016), including all fields of study. First time student numbers have not been published for private HEls. Comparing the total numbers of enrolment (second or higher year students), 975,000 students for public HEls and 167,000 students for private HEls are mentioned.

Naked enrolment numbers alone don't fully cover the picture. When comparing the statistics with Flanders (a federal state in Belgium), the magnitude of the skill shortage in South Africa becomes evident. In Flanders, $41 \%$ of the population between 25-64 years old has a degree of tertiary education (Statistiek Vlaanderen, 2019). In contrast, from the same age group in South Africa, only $12 \%$ has a degree of higher education (DSRSA, 2017).

In general, more than half $(51 \%)$ of the youths in South Africa indicate that they have no money to pay for tuition at tertiary educational facilities (Dept. of Statistics, 2019). Combined with the low level of secondary level schools and low graduation numbers, this makes a very small pool of students for the HEls to recruit from.

Alongside not having the money to pay for tuition fees, the younger people are expected to work in order to raise money for their families. Even though South Africa is seen as an average income country, $56 \%$ of the population lives in poverty (less than R992 or EUR 60 per person per month) and $25.2 \%$ in absolute poverty (less than R441 or EUR 27 per person per month). A bread costs around EUR 1 on average in South Africa, indicating the stringing income shortage. This lack of income is for a large amount of young people the major factor of not starting or completing their studies: it is unthinkable for them to study for multiple years.

These are major demographic and economic impacts on the recruitment process. The quality management system must make sure that the correct students, how few they might be, are starting their studies at the institution. The impacts on the recruitment processes are far larger than in Belgium, where a large part of the population has the opportunity to start a tertiary educational programme.

Due to these factors, the processes of Belgium Campus in South Africa must incorporate the support of the industry, where the students get funded by a company. This approach leads to direct employment upon completion of the studies. However, it also causes a potential for industrial interference with the curriculum, or early retirement from the study trajectory (due to several factors, which are elaborated in Section 4.2). 
From the recruitment point of view, the HEls are also supported from the government in South Africa, by the "Broad-based Black Economic Empowerment". This stimulates companies to recruit, train and employ the minorities and disadvantaged groups in the society. This policy provides extra potential for recruitment, but it is not able to compensate for the above mentioned challenges. Furthermore, it is not capable of providing the necessary inflow of students that South Africa needs.

Quality processes aim, for student recruitment, to get the right students in the right place, and keep them there. Different influences (industrial or governmental) aim to have a positive impact on the recruitment processes of the $\mathrm{HEI}$.

\subsection{Student retention}

After recruiting the students, it is obvious that specific mechanisms and processes are needed to keep them within their study trajectory. Due to the already mentioned demographic factors, keeping the students is not that straightforward.

The first factor to be considered is two-fold: young people are encouraged to find a job to provide for their families, and the industry has major difficulties finding the right people due to the skill shortage. However, there is a major contradiction when stating: 'a shortage in skilled employees' and 'an excess of unemployed, unskilled people' (Gianchandani, 2018). Working together with industry to recruit, retain and employ the students, is paramount. However, the economic setting in South Africa makes that students are often recruited early-on by companies to come and work for them. Even after one or two years of study (and thus before graduation), students are tempted to abandon their studies.

This must be countered by providing enough reason for the students to keep up their educational career. At this point, appropriate processes are needed within the QMS, such as providing student facilities, providing encouragement (individual learning programs) and perhaps most of all, comforting a student into staying, which is a direct result of the close relationship between teaching staff and students.

Student facilities are a well known concept in Europe. This is the same in South Africa, and especially for Belgium Campus. The general facilities such as student housing, extended learning duration with individual programmes and extra time on the exam, are implemented. However, due to the very diverse student population, the institution must go beyond these "simple" facilities.

These "extended" facilities are provided for the students with specific special needs, such as the different minority groups and the deaf community. These diverse groups have got a wide variety of supplemental specialized support to keep them within their studies. A perfect example was the implementation of a whole supporting structure for the deaf community amongst their students. By providing sign language translators and other specific facilities, these students actually have a chance of making it through their studies, and graduating with a degree. Literature also indicates that special processes should be in place when targeting facilities towards minority groups (KingBerry et al., 2018).

Another example of a student "facility", is the obligation of wearing a uniform. This helps to reduce the differences between different ethnic groups and incomes. Reduced differences (even in ap- 
pearance) help to stimulate the creation of a true class group. The less the student has the feeling he/she "stands out" in a certain way, the smaller the chances of abandoning his/her studies are.

As could be concluded from the panel conversations the external commission had with the students during the on-site visit, the students are very satisfied. The institution already facilitated a significant number of student's initiatives, but neither the students, nor the HEI, think they have reached the full potential with regard to student retention yet. Further cooperation between all stakeholders could increase social integration even more. An example is to improve the social fun-factor, through extracurricular and spare time activities. These activities encourage community building and the sense of unity. This is a prerequisite to achieve a maximally effective academic integration that will help students to get the most out of their student life. Furthermore, it provides a significant added value the student and his later career: stronger human capital networks, personal growth as a person and as a citizen.

Of course, a drop-out rate will always exist. Reducing student drop-out rates in higher education is a challenge faced by institutions all over the world. The existence of drop out was evidenced by the success rates in the self-evaluation report and confirmed during the panel conversations. Causes for drop-out are manifold and often external to the institution itself (e.g. labour market pressure, financial constraints, skills gap between secondary and tertiary education). Further analyses, e.g. a drop-out survey, can give insight on the main reasons for drop-out and encourage the HEls to reflect on the best ways to cope with this problem. Interestingly, students of Belgium Campus who interrupted their studies, do sometimes return after a couple of years to finish their studies. This shows how much stakeholders recognize the value of the awarded degree in the long term. This also reinforces the fact that financial problems are often the major cause for the drop-out within South African HEls.

Another crucial factor to student retention is the way the students and staff are able to communicate. It was obvious during the site visit, that the students and staff highly appreciate the open door policy. Nevertheless, the current growth of the institution can compromise the ability for open communication. Alongside the students' different background, ethnicity and cultures, the intercultural staff team increases the ability to strengthen the open door policies.

Due to the current growth of the institution, one must be aware of the vulnerability of those open door policy and direct communication lines. Even in Belgium, large campuses and multi-campus institutions are vulnerable to a hierarchical structure hindering student to staff communications. This current strength is easily lost unless appropriate action is taken to ensure short and direct communication lines, in spite of a distant campus arrangement and increasing student numbers. Especially the new multi-campus model (supporting the current growth) has to ensure the student's well-being on the campuses.

Furthermore, the distance between the campuses is substantial. The third and newest campus is $1,100 \mathrm{~km}$ away from the main campus. This will also have an influence on how the quality management structures work. On the one hand, all campuses should keep up the overall values and policies that the institution stands for. On the other hand, due to the sheer distance, other demographic influences will surely leave their mark. When travelling in Europe, a thousand kilometre travel also results in changed cultures and/or religions. Even the very composition of the population at the other side of South Africa might be substantially different. This will also result in a need for adapted processes of recruiting and retaining the students. Although being part of the same 
institution, the different campuses will have to operate independently and will differ in their own ways while staying true to institution's values. This multi-campus model is also implemented at the universities employing the members of the external commission performing the audit (Vermeersch, 2015), with an official accreditation audit held at six different campuses simultaneously.

The three individual campuses in South Africa provide a larger geographical area to recruit students. More campuses, and more students, will increase the number of professional graduates that South Africa so craves. Nevertheless, the campuses should stay "reasonably" small, ensuring the open door policy and short communication lines. The same applies to the Flanders' Engineering landscape: multiple campuses with their own individual focus points, while maintaining the link with the coordinating institution.

\subsection{Student Employability}

After recruiting and training a student, it is important to make sure that he/she has all the competences and skills needed to find a job, which in most cases is the main goal of an educational programme. Employability is a key factor within education and it is a good indication of the quality of the educational programmes offered by an institution.

Belgium Campus has a track record where a 100\% employment rate of their graduates is established. When graduating, the students are immediately employable in industry. The curriculum has been drafted with employability as a major driving factor.

The students are trained to show sound character and attitude, and they are able to organise their further academic development. Furthermore, they are trained to become leaders in their professional fields and communities. The institute aims to connect knowledge with skills and develop a positive personality and a growth mindset to equip the 21 st-century learner.

These traits are very much appreciated by the employing parties. The fact that Belgium Campus has a $100 \%$ employment rate, is reinforced by the fact that students are recruited while still studying. This result is a testimony to the solid foundations created by the institution. Even in a troubling and hard environment within the South African context this result is obtained. This is true empowerment of the people and their communities.

This also shows that the vision and the actual quality of the programme (and institution as a whole) go far beyond simple employment. During the course of the programme, students learn to work together, form a group, develop skills and knowledge that have a direct impact on the population and communities of South Africa.

Even though a "perfect" track record is observed, the adverse role of the industry should never be underestimated, especially in the demographical context of South Africa. In Belgium and Europe, the companies are well aware that university colleges and universities are delivering welleducated students of a sufficiently high level. In South Africa however, the skill shortage is so stringent that students are temped to leave the HEI to go to work, long before their education is finished. Furthermore, due to the specific skills required, companies are lobbying for specific skill sets to be included in different curricula. The academic freedom must be defended. One should aim for the "perfect balance" between teaching the student the generic and innovative basics of technology, and providing the specific technologies and soft skills that the industry needs. From the audit, Belgium Campus is judged to be well equipped to deal with this useful but delicate bal- 
ance. This balance with regard to industrial influence is also a factor to be considered within the QMS. The dynamics of this balance strongly depend on demographical and societal factors.

\section{Conclusions}

This paper started by introducing the generally accepted and applied European Standards and Guidelines for Quality Assurance in Higher Education (ESG). The ESG provide guidance on how to perceive quality. The perception of quality can be influenced significantly by societal and demographic factors. These external factors become increasingly important for higher educational institutions (HEls), since these factors affect the way the HEls need to operate with regard to student recruitment, student retention and student employability.

Within an intercontinental context of collaboration between educational institutions, it is not straightforward to map quality and quality management processes one-on-one. Specific processes used in Belgium/Europe might take an entirely other form in South Africa. Although they look fundamentally different, differences do not (always) imply a different quality. Approaches which function perfectly for HEls in South Africa, might not be well suited for European HEls and vice versa. Examples are the sponsorship of students by the industry, the fulfilment of the needs of the Deaf community or the specific aid the government provides for minority groups ("Broad Based Black Economic Empowerment"). These circumstances call for specifically tailored student recruitment processes.

It should be noted that not all peculiarities in the QMS are due to demographic or societal factors. Low quality is still possible. Bad approaches obviously must be tracked and resolved. No external factors are allowed to be an excuse for low quality processes.

This paper explicitly handled the peculiarities within the QMS that are due to external demographic and societal factors. They do not represent low quality processes, although external people might have wrong perception of a quality related problem.

All peculiarities of the QMS of Belgium Campus in South Africa have been tracked and traced during the audit, resulting in an accurate pinpointing of the causes and solutions that were implemented in the QMS. The audit led to specific insights in how demographic and societal factors influence students' life-cycle elements, as presented in this paper.

By distinguishing three phases in a student's life-cycle within an institution, the authors highlighted the different factors that are influencing the QMS. The impact of demographic, societal and economic factors was considered. Although the conclusions are valid for a South African institution, the demographical conclusions and impacts are also useful in Europe, where a more and more diverse society is emerging. Educational institutions should be able to handle this diversity, assisted by specific and targeted quality management processes. International collaboration widens the views on these matters and can provide guidance on how to deal with diversification.

In essence, the awareness of the different factors, leads to a better performing QMS and therefore, a more accurate quality perception of the institution. Both lead to a stronger position on the national and international field of research and education. Students especially benefit from the specific demographic considerations made by an institution. Due to HEls' highly influential role 
within the society, a better awareness also results in students that are more easily employable and have appropriate tools and knowledge to increase the level of both industry and society. 


\section{References}

Department of Higher Education and Training (DHET) Republic of South Africa. (2016). Statistics on PostSchool Education and Training in South Africa. [Online]. Available at: https://www.jet.org.za/news/dhet-releases-statistics-on-post-school-education-and-training-in-southafrica-2016 (Accessed 25 April 2019).

Department of Statistics Republic of South Africa (DSRSA). Education. (2019). [Online]. Available at: http://www.statssa.gov.za/?page id=737\&id=4 (Accessed: 16 April 2019).

Department of Statistics Republic of South Africa (DSRSA). Education Series Volume III: Educational Enrolment and Achievement, 2016. (2017). [Online]. Available at: http://www.statssa.gov.za/publications/Report\%2092-01-03/Report\%2092-01-032016.pdf

Federale Overheidsdienst Binnenlandse Zaken (IBZ), Bevolkingscijfers per provincie en per gemeente op 1 januari 2019. [Online]. Available at: https://www.ibz.rrn.fgov.be/nl/bevolking/statistieken-vanbevolking/

Flemish Government. (No date). Hoger Onderwijs in Cijfers. [Online]. Available at: http://dataonderwijs.vlaanderen.be/documenten/bestand.ashx?id=11615 (Accessed: 16 April 2019)

Gianchandani, P. (2018) 'Why companies invest in vocational training \& apprenticeships', Proceedings of EDULEARN18 Conference 2nd-4th July 2018, Palma, Mallorca, Spain, pp. 9628-9636.

Jacobs, E. (2018) Self-evaluation report 2018 - Belgium Campus ITversity, October 2018. [Online]. Available at: https://www.belgiumcampus.ac.za/PDF/Downloads/Self-evaluation\%20report\%202018.pdf

King-Berry, A., Boone, R., Johnson, N.M., Kalunga, R. (2018) 'African American students with disabilities: beneficiaries of the legacy or disproportionality?', Proceedings of EDULEARN18 Conference 2nd-4th July 2018, Palma, Mallorca, Spain, pp. 8677-8683.

Popa, D., Moraru, G.-M. (2019) 'Universities as promotors of sustainable development', Proceedings of EDULEARN19 Conference, 1st-3rd July 2019, Palma, Mallorca, Spain, pp. 4405-4409.

Remaud, B., Berbers, Y., Jolly, A.-M., Nolland, J. (2017) 'Accreditation of Flemish Civil Engineers programmes (2016): an experience of cross-border Quality Assurance', Proceedings of the 45th Annual SEFI Conference, 18-21 September 2017, Azores, Portugal, pp. 669-676.

Standards and Guidelines for Quality Assurance in the European Higher Education Area (ESG). (2015). Brussels, Belgium.

Statistiek Vlaanderen, Bevolking naar onderwijsniveau (scholingsgraad), May 2019. [Online]. Available at: https://www.statistiekvlaanderen.be/bevolking-naar-onderwijsniveau-scholingsgraad

Supernak, J. (2018) 'Striving for excellence of undergraduate education: is meeting accreditation criteria enough?', Proceedings of EDULEARN18 Conference 2nd-4th July 2018, Palma, Mallorca, Spain, pp. 7654-7663.

Vermeersch, J. (2015) 'Coaching stakeholders involved in external program evaluations', Proceedings of the 43th Annual SEFI Conference, June 29 - July 2, 2015, Orléans, France, paper 67742. 